\title{
An international multicenter study comparing arrhythmia prevalence between the intracardiac lateral tunnel and the extracardiac conduit type of Fontan operations
}

\author{
Seshadri Balaji, MBBS, MRCP (UK), PhD, ${ }^{a}$ Ankana Daga, MD, ${ }^{b}$ David J. Bradley, MD, \\ Susan P. Etheridge, MD, Ian H. Law, MD, ${ }^{\mathrm{e}}$ Anjan S. Batra, MD, ${ }^{\mathrm{f}}$ Shubayan Sanatani, MD, ${ }^{\mathrm{g}}$ \\ Anoop K. Singh, MD, ${ }^{\mathrm{h}}$ Kelly K. Gajewski, MD, ${ }^{\mathrm{i}}$ Sabrina Tsao, MD ${ }^{\mathrm{j}}$ Harinder R. Singh, MD, ${ }^{k}$ \\ Svjetlana Tisma-Dupanovic, MD, ${ }^{1}$ Shigeru Tateno, MD, ${ }^{\mathrm{m}}$ Motoki Takamuro, MD, ${ }^{\mathrm{m}}$ \\ Hiromichi Nakajima, MD, ${ }^{\mathrm{n}}$ Jolien W. Roos-Hesselink, MD, PhD, ${ }^{\circ}$ and Maully Shah, MBBS ${ }^{\mathrm{b}}$
}

Objective: The study objective was to determine whether the extracardiac conduit Fontan confers an arrhythmia advantage over the intracardiac lateral tunnel Fontan.

\begin{abstract}
Methods: This multicenter study of 1271 patients compared bradyarrhythmia (defined as need for pacing) and tachyarrhythmia (defined as needing antiarrhythmic therapy) between 602 patients undergoing the intracardiac Fontan and 669 patients undergoing the extracardiac Fontan. The median age at the time of the Fontan procedure was 2.1 years (interquartile range, 1.6-3.2 years) for the intracardiac group and 3.0 years (interquartile range, 2.4-3.9) for the extracardiac group $(P<.0001)$. The median follow-up was 9.2 years (interquartile range, $5-12.8)$ for the intracardiac group and 4.7 years (interquartile range, 2.8-7.7) for the extracardiac group $(P<.0001)$.
\end{abstract}

Results: Early postoperative ( $<30$ days) bradyarrhythmia occurred in 24 patients $(4 \%)$ in the intracardiac group and 73 patients $(11 \%)$ in the extracardiac group $(P<.0001)$. Early postoperative $(<30$ days $)$ tachyarrhythmia occurred in 32 patients $(5 \%)$ in the intracardiac group and 53 patients $(8 \%)$ in the extracardiac group $(P=$ not significant). Late ( $>30$ days) bradyarrhythmia occurred in 105 patients $(18 \%)$ in the intracardiac group and 63 patients $(9 \%)$ in the extracardiac group $(P<.0001)$. Late $(>30$ days $)$ tachyarrhythmia occurred in 58 patients $(10 \%)$ in the intracardiac group and 23 patients $(3 \%)$ in the extracardiac group $(P<.0001)$. By multivariate analysis factoring time since surgery, more patients in the extracardiac group had early bradycardia (odds ratio, 2.9; $95 \%$ confidence interval, 1.8-4.6), with no difference in early tachycardia, late bradycardia, or late tachycardia.

Conclusions: Overall arrhythmia burden is similar between the 2 groups, but the extracardiac Fontan group had a higher incidence of early bradyarrhythmias. There was no difference in the incidence of late tachyarrhythmias over time between the 2 operations. Therefore, the type of Fontan performed should be based on factors other than an anticipated reduction in arrhythmia burden from the extracardiac conduit. (J Thorac Cardiovasc Surg 2014;148:576-81)

From the Department of Pediatrics, ${ }^{\text {a }}$ Oregon Health \& Science University, Portland, Ore; Department of Cardiology, ${ }^{\text {b }}$ Children's Hospital of Philadelphia, Philadelphia, Pa; Department of Cardiology, ${ }^{\mathrm{c}}$ CS Mott Children's Hospital, Ann Arbor, Mich; Department of Cardiology, ${ }^{\mathrm{d}}$ Primary Children's Medical Center and University of Utah, Salt Lake City, Utah; Department of Pediatrics, ${ }^{\text {e }}$ University of Iowa, Iowa City, Iowa; Department of Pediatrics, ${ }^{\mathrm{f}}$ University of California, Irvine, Calif; Department of Cardiology, ${ }^{g}$ Children's Hospital, Vancouver, British Columbia, Canada; Department of Cardiology, ${ }^{\text {h }}$ Children's Hospital of Wisconsin, Milwaukee, Wis; Department of Pediatrics, ${ }^{i}$ Medical University of South Carolina, Charleston, SC; Department of Cardiology, ${ }^{\mathrm{j}}$ Children's Memorial Hospital, Chicago, Ill; Department of Cardiology, ${ }^{\mathrm{k}}$ Children's Hospital of Michigan, Detroit, Mich; Department of Cardiology, ${ }^{1}$ Mercy Children's Hospital, Kansas City, Mo; Department of Pediatric Cardiology, ${ }^{\mathrm{m}}$ Children's Hospital \& Cardiovascular Center, Chiba, Japan; Department of Pediatric Cardiology, ${ }^{\mathrm{n}}$ Hokkaido Medical Center, Hokkaido, Japan; and Department of Cardiology, ${ }^{\circ}$ Erasmus University, Rotterdam, The Netherlands.

This study was supported by Grant 28910-7298110612 from the Children's Hospital of Philadelphia.

Disclosures: Authors have nothing to disclose with regard to commercial support.

Received for publication April 19, 2013; revisions received Aug 13, 2013; accepted for publication Aug 22, 2013; available ahead of print Oct 29, 2013.

Address for reprints: Maully Shah, MBBS, The Cardiac Center, The Children's

Hospital of Philadelphia, 3400 Civic Center Blvd, Philadelphia, PA 19104

(E-mail: shahm@email.chop.edu).

0022-5223/\$36.00

Copyright (c) 2014 by The American Association for Thoracic Surgery

http://dx.doi.org/10.1016/j.jtcvs.2013.08.070
Earn CME credits at

http://jtcvs.com/cme/home

The Fontan operation, first described in the early 1970s, revolutionized the management of children with single ventricles. ${ }^{1}$ Since inception, the procedure has undergone numerous modifications. Chief among these was the development of the intracardiac lateral tunnel (ILT). First described by de Leval and colleagues, ${ }^{2}$ the ILT improved surgical outcomes compared with the original atriopulmonary connection (APC) and was therefore generally adopted. Soon thereafter, Marcelletti and colleagues ${ }^{3}$ described the extracardiac conduit (ECC) modification, which, although similar to the ILT, bypasses the right atrium with a conduit from the inferior vena cava to the pulmonary artery.

Arrhythmias, both bradycardia (mainly sinus node dysfunction [SND]) and, more important, tachyarrhythmias (primarily supraventricular tachycardia [SVT] due to atrial 


\author{
Abbreviations and Acronyms \\ $\mathrm{AFL}=$ atrial flutter \\ $\mathrm{APC}=$ atriopulmonary connection \\ $\mathrm{ECC}=$ extracardiac conduit \\ $\mathrm{ECG}=$ electrocardiography \\ IART $=$ incisional atrial reentrant tachycardia \\ ILT = intracardiac lateral tunnel \\ $\mathrm{IQR}=$ interquartile range \\ $\mathrm{OR}=$ odds ratio \\ $\mathrm{SD}=$ standard deviation \\ SND $=$ sinus node dysfunction \\ SVT $=$ supraventricular tachycardia
}

flutter [AFL] or incisional atrial reentrant tachycardia [IART]) have been a challenging problem in patients undergoing the Fontan..$^{4-6}$ Bradyarrhythmias are thought to be primarily due to damage to the sinus node or its arterial supply. Tachyarrhythmias are thought to be due to suture lines and pressure and volume overload of the right atrium. Although early bradyarrhythmias and tachyarrhythmias can complicate the immediate postoperative management and increase the hospital length of stay, they are often transient. In the long-term, bradyarrhythmias can be managed by pacemaker implant. However, tachyarrhythmias in this patient population can be a major cause of morbidity and mortality and hard to manage.

The ILT resulted in less late tachyarrhythmia compared with the APC. ${ }^{4-6}$ Marcelletti and colleagues ${ }^{3}$ proposed that the ECC would be even less arrhythmogenic because it bypasses the right atrium. ${ }^{7}$ To date, most studies comparing the ILT with the ECC have been cross-sectional rather than longitudinal and have been inconclusive in determining whether the ECC provides a long-term arrhythmia benefit compared with the ILT. ${ }^{8-17}$

To better evaluate the incidence of arrhythmia in the current era, a multicenter international retrospective study was designed to examine the prevalence and potential difference in early ( $\leq 30$ days) and late ( $>30$ days) bradyarrhythmias and tachyarrhythmias after the ILT and ECC operations.

\section{MATERIALS AND METHODS}

This study was a retrospective cohort study, with The Children's Hospital of Philadelphia serving as the data coordinating center. The study received approval by an institutional review board at each participating institution. All members of the Pediatric and Congenital Electrophysiology Society were contacted and invited to participate in the study.

\section{Study Population}

Subjects were identified through a database search and included all patients who underwent an ILT or ECC between January 1, 1998, and January 1, 2008. Patients were excluded from the study if (1) an arrhythmia was documented before their ILT or ECC and (2) follow-up after ILT or ECC was less than 1 year or (3) a previous APC had been performed. A small group of patients $(n=56)$ at one institution were part of a study of patients with ILT randomized to receive or not receive additional incisions within the right atrium, which may prevent the development of postoperative arrhythmias. We analyzed our data with and without this subset of patients and did not identify any difference; therefore, the data presented include these 56 patients.

\section{Medical Record Review}

Standardized data forms were used to extract pertinent data from medical records regarding patient demographics, cardiac anatomic diagnosis, type of surgical repair, electrocardiography (ECG) and ambulatory Holter monitor findings, and presence of rhythm disturbances. When rhythm disturbances were documented, the onset, type, and treatment for the rhythm disturbance were noted.

\section{Rhythm Analysis}

Relevant rhythm documentation was reviewed at the individual institutions. If bradyarrhythmias were documented, they were classified as SND or atrioventricular block. If tachyarrhythmias were present, they were classified as SVT or ventricular tachycardia. For the purpose of this study, bradycardia was considered significant if it required pacing. SVT was defined as a rhythm disturbance originating above the ventricles resulting in a fast heart rate causing death or requiring acute or chronic therapy, such as pharmacotherapy, antitachycardia pacing, direct current cardioversion or defibrillation, catheter ablation, or arrhythmia surgery. We did not subcategorize the mechanism of SVT as being due to AFL/IART.

\section{Surgical Procedures}

Patients were divided into 2 Fontan groups according to the following: (1) If an external conduit connected the inferior vena cava to the pulmonary artery, patients were classified as having the ECC type of Fontan. (2) If an intra-atrial tunnel connected the inferior vena cava to the pulmonary artery, patients were classified as having the intracardiac lateral tunnel (ILT) type of Fontan. In patients with unusual anatomy, such as heterotaxy syndromes, regardless of modifications used in their surgery, we designated patients using the listed criteria. No patients were excluded from the analysis because of modifications to their surgery.

Surgeries preceding the Fontan operation and the presence or absence of Fontan fenestration were documented. The early postoperative period was defined as the time period 30 days or less after Fontan surgery. The late postoperative period was defined as the period more than 30 days after Fontan surgery.

\section{Study End Points}

The primary end points of this study were (1) documented bradyarrhythmia requiring pacing, either temporary or permanent (any patient who received temporary or permanent pacing in the early postoperative period was noted), and (2) documented SVT causing death or clinical symptoms requiring management with commonly used therapeutic options.

\section{Statistical Methods}

Descriptive statistics were calculated for demographic and descriptive data, reported as median with interquartile range (IQR) for quantitative variables and number and percentage for qualitative variables. Demographic and clinical variables were compared between the 2 groups (ILT vs ECC) using the Mann-Whitney $U$ test, Pearson chi-square test, or Fisher exact test as appropriate.

Multivariate logistic regression models were used to assess the relationship between the type of Fontan (bidirectional Glenn, early arrhythmia, late arrhythmia, hemi-Fontan, and fenestration) adjusting for age at Fontan.

Kaplan-Meier survival curves were generated for time to first late bradyarrhythmia and time to first late tachyarrhythmia. Multivariate Cox regression models were used to assess whether there was a difference between the 2 groups adjusting for age at Fontan. In addition, we also 
TABLE 1. Patient characteristics

\begin{tabular}{|c|c|c|c|}
\hline Variable & ILT (\%) & $\operatorname{ECC}(\%)$ & $P$ value \\
\hline $\mathrm{N}$ & 602 & 669 & NA \\
\hline Male & $231(60)$ & $286(60)$ & NS \\
\hline Dominant RV & $292(49)$ & $266(40)$ & .007 \\
\hline Dominant LV & 165 (27) & $219(33)$ & NS \\
\hline Other SV & 145 (24) & $184(28)$ & NS \\
\hline Norwood* & 298 & 296 & NS \\
\hline Shunts $\dagger$ & 190 & 233 & NS \\
\hline PA band & 55 & 66 & NS \\
\hline Other & 21 & 22 & NS \\
\hline No surgery & 38 & 52 & NS \\
\hline Hemi-Fontan & $339(69)$ & $24(6)$ & $<.0001$ \\
\hline Bidirectional Glenn & $58(12)$ & $296(70)$ & $<.000$ \\
\hline Fenestration & $422(71)$ & $468(70)$ & NS \\
\hline $\begin{array}{l}\text { Age at surgery } 1 \text { (d) } \\
\text { (median, IQR) }\end{array}$ & $7(4-30)$ & $8(3-30)$ & NS \\
\hline $\begin{array}{l}\text { Age at surgery } 2(\mathrm{mo}) \\
\text { (median, IQR) }\end{array}$ & $6.7(5-11)$ & $6.6(5-10)$ & NS \\
\hline $\begin{array}{l}\text { Age at Fontan (y) } \\
\text { (median, IQR) }\end{array}$ & $2.1(1.6-3.2)$ & $3.0(2.4-3.9)$ & $<.000$ \\
\hline $\begin{array}{l}\text { Age at last FU }(\mathrm{y}) \\
\text { (median, } \mathrm{IQR})\end{array}$ & $11.8(7.7-16.5)$ & $7.9(5.4-10.8)$ & $<.0001$ \\
\hline $\begin{array}{c}\text { FU since Fontan }(y) \\
\text { (median, } \mathrm{IQR})\end{array}$ & $9.2(5-12.8)$ & $4.7(2.8-7.7)$ & $<.000$ \\
\hline
\end{tabular}

$\overline{E C C}$, Extracardiac conduit; $F U$, follow-up; $I L T$, intracardiac lateral tunnel; $I Q R$, interquartile range; $L V$, left ventricle; $N A$, not available; $N S$, not significant; $P A$, pulmonary artery; $R V$, right ventricle; $S V$, single ventricle. *Includes all Norwood-type operations. $\dagger$ Includes all varieties of systemic to pulmonary shunts.

performed multivariate Cox regressions adjusting for patient clustering by site with robust sandwich covariance matrix estimation in an effort to see whether patient clustering by center had an effect on results.

\section{RESULTS \\ Patients}

A total of 1500 patient records from 14 tertiary care cardiac centers (10 US, 1 Canadian, and 3 international centers [2 from Japan and 1 from The Netherlands]) were screened for study participation. There were 1271 patients $(84.7 \%)$ identified as eligible and included in the study. Of these, $602(47 \%)$ were in the ILT group and $669(53 \%)$ were in the ECC group. Table 1 outlines the demographic aspects of the 2 groups. There was no difference in gender between the 2 groups (Table 1). The ILT group had a slightly higher proportion of patients with a dominant right ventricle and a slightly higher incidence of the Norwood-type operation as the initial surgery. Operations before the stage 1 Fontan repair (ie, bidirectional Glenn or hemi-Fontan) were broken down into Norwood type procedures (including Sano modification and Damus-Kaye-Stansel operations), all shunts (mostly Blalock-Taussig shunts but also a few central and other types of shunts), pulmonary artery bands and other surgeries (including coarctation repair without pulmonary artery band), repair of anomalous pulmonary venous drainage, valvuloplasty surgery, and a small number in each group who had undergone no prior surgery.
The majority of the patients in the ILT group had previously undergone the hemi-Fontan operation, whereas most patients in the ECC group had undergone the bidirectional Glenn operation. Patients in the ILT group were younger at the time of the Fontan (median, 2.1 years; IQR, 1.6-3.2 vs median, 3.0; IQR, 2.4-3.9; $P<.0001)$ and older at the time of last follow-up (median, 11.8 years; IQR, 7.7-16.5 vs median, 7.9; IQR, 5.4-10.8; $P<.0001$ ). The interval between surgery and last follow-up was greater in the ILT group (median, 9.2; IQR, 5-12.8; vs median, 4.7; IQR, 2.8-7.7; $P<.0001)$.

There were a total of 110 patients with heterotaxy $(8.6 \%$ of the total), and of these $70(64 \%)$ underwent an ECC and the remaining $(30,36 \%)$ had an intracardiac tunnel.

\section{Early Postoperative Arrhythmia}

Bradycardia requiring pacing occurred in 97 patients $(8 \%)$ in the early postoperative period. Of these, 71 patients $(73 \%)$ had only temporary pacing and permanent pacemakers were implanted in $26(27 \%$ of the bradycardia subgroup and $2 \%$ of entire cohort). Of the 26 patients who had permanent pacemakers, $9(35 \%)$ were in the ILT group and $17(65 \%)$ were in the ECC group (Table 2).

Of the 71 patients who had only temporary pacing in the early postoperative period, $7(10 \%)$ underwent implantation of a permanent pacemaker in the late postoperative period.

Tachyarrhythmias occurred early after surgery in 32 patients $(5 \%)$ in the ILT group and 53 patients $(8 \%)$ in the ECC group ( $P=$ not significant). When bradyarrhythmias and tachyarrhythmias were combined, the burden of arrhythmias in the early postoperative period was 56 in the ILT group and 126 in the ECC group $(P=.0003)$.

By multivariate comparison (performed using logistic regression with adjustment for age at Fontan) (Table 3) of arrhythmias in the early period, the ILT group was less likely to be paced compared with the ECC group (odds ratio [OR], 0.35; confidence interval [CI], 0.22-0.57; $P<.0001$ ), but the incidence of early tachyarrhythmia did not differ between groups (OR, 0.69; CI, 0.43-1.09; $P=.1$ ).

\section{Late Postoperative Arrhythmias}

On univariate analysis (Table 2), both bradycardia and tachycardia were more common in the ILT group compared with the ECC group. Subdividing the tachycardia group into

TABLE 2. Results of univariate analysis

\begin{tabular}{lccc}
\hline Variable & ILT n (\%) & ECC n (\%) & P value \\
\hline Early bradycardia & $24(4)$ & $73(11)$ & $<.0001$ \\
Early tachycardia & $32(5)$ & $53(8)$ & .063 \\
Late bradycardia & $105(18)$ & $63(9)$ & $<.0001$ \\
Late tachycardia & $58(10)$ & $23(3)$ & $<.0001$ \\
\hline
\end{tabular}

$E C C$, Extracardiac conduit; ILT, intracardiac lateral tunnel. 
TABLE 3. Results of multivariate analysis

\begin{tabular}{lcll}
\hline Variable ILT vs ECC & Odds ratio/hazard ratio* & $\mathbf{9 5} \%$ CI & $\boldsymbol{P}$ value \\
\hline Early bradycardia & 0.35 & $0.22-0.57$ & $<.0001$ \\
Early tachycardia & 0.69 & $0.43-1.09$ & .1 \\
Late bradycardia & 1.26 & $0.76-2.1$ & .38 \\
Late tachycardia & 1.32 & $0.74-2.33$ & .35 \\
\hline
\end{tabular}

$C I$, Confidence interval; $E C C$, extracardiac conduit; $I L T$, intracardiac lateral tunnel. * Odds ratios from multivariate logistic regression are reported for early bradycardia and early tachycardia; hazard ratios from Cox regression are reported for late bradycardia and late tachycardia. Age and gender were adjusted in the multivariate analysis.

those requiring acute versus chronic therapy, tachyarrhythmias requiring acute therapy (mostly direct current cardioversion) were identified in 44 patients $(7 \%)$ in the ILT group compared with 13 patients $(2 \%)$ in the ECC group $(P<.0001)$. Tachyarrhythmias requiring chronic therapy were observed in 38 patients $(6 \%)$ in the ILT group and 17 patients $(3 \%)$ in the ECC group. The differences between ILT and ECC observed on univariate analysis were shown to be due to the longer follow-up in the ILT group when multivariate analysis was performed (Kaplan-Meier graphs, Figures 1 and 2).

On multivariate analysis (Table 3), there were 573 patients in the ILT group and 566 patients in the ECC group with data available for multivariate analysis and creation of Kaplan-Meier graphs for bradyarrhythmia. Data were available for 576 patients in the ILT group and 570 patients in the ECC group for analysis of late tachyarrhythmia.

There was no difference in late postoperative bradycardia or tachycardia between the 2 groups. Figures 1 and 2 show the Kaplan-Meier curves for late bradycardia and tachycardia.

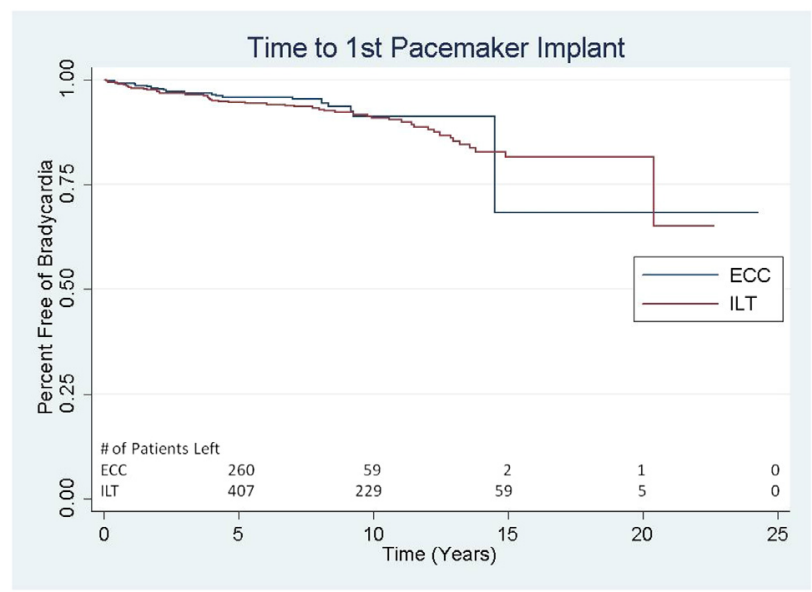

FIGURE 1. Kaplan-Meier curve for late bradyarrhythmia showing time to first bradycardia. $P=.41$; incidence for ILT is $1.1 \%$ per year with 5 -year cumulative probability of 5.4\% (standard deviation [SD], $1.0 \%$ ); incidence for ECC is $0.8 \%$ per year with 5 -year cumulative probability of $4.2 \%$ (SD, $0.9 \%$ ). ECC, Extracardiac conduit; ILT, intracardiac lateral tunnel.

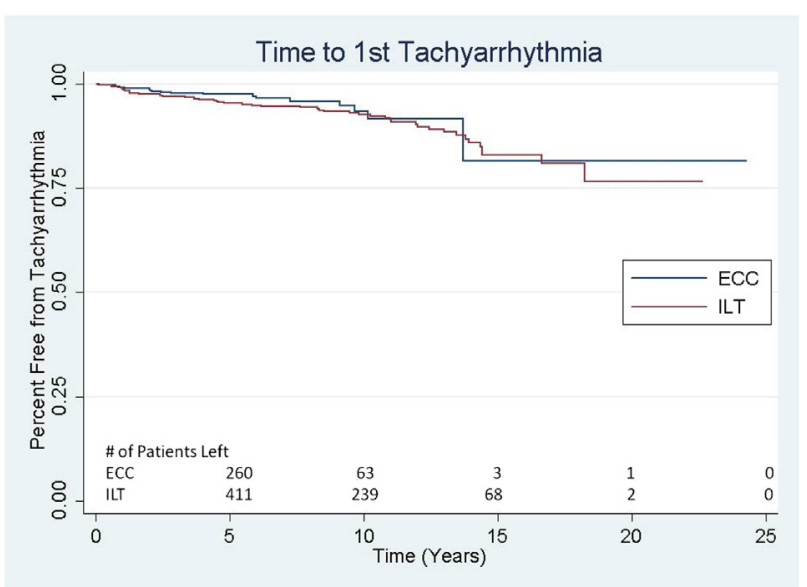

FIGURE 2. Kaplan-Meier curve for late tachyarrhythmia showing time to first tachycardia. $P=.31$; incidence for ILT is $1.0 \%$ per year with 5 -year cumulative probability of $4.4 \%$ ( $\mathrm{SD}, 0.9 \%$ ); incidence for ECC is $0.6 \%$ per year with 5 -year cumulative probability of $2.6 \%(\mathrm{SD}, 0.9 \%$ ). $E C C$, Extracardiac conduit; ILT, intracardiac lateral tunnel.

Early arrhythmia did not predict late arrhythmia (ILT vs ECC OR, 1.28; CI, 0.89-1.84) in the whole group and in either subgroup. We observed no "center effect" on the results because of patients clustering in one or few centers.

\section{DISCUSSION}

Then main findings of this study were that apart from the ECC being associated with a higher incidence of early SND, the incidence of late arrhythmias (especially late SVT) was similar for the ILT and the ECC when factoring in the duration of follow-up.

Arrhythmias are one of the most frequent and troublesome complications encountered in the population undergoing the Fontan, occurring in the early postoperative period and throughout long-term follow-up. These arrhythmias often are hemodynamically significant, challenging to control, and represent a significant source of morbidity and mortality in this population. When de Leval and colleagues ${ }^{2}$ proposed the ILT, their primary purpose was to improve hemodynamics. However, one of their hopes was that it would decrease the arrhythmia burden compared with the earlier APC Fontan. ${ }^{2}$ This has indeed proven to be the case. ${ }^{4-6}$ However, although it reduces the incidence, arrhythmias have remained sequelae of the ILT. $^{4-6}$ The ECC was proposed by Marcelletti and colleagues ${ }^{3}$ as a way of completely bypassing the right side of the heart, with the hope that this technique could reduce the risk of SVT. ${ }^{7}$ Again, despite these modifications, arrhythmias persist. ${ }^{8-17}$

Factors shown to be associated with arrhythmias in patients undergoing the Fontan include the extent of surgery close to the sinus node and its artery, the extensive suture lines in the atrium, and the presence of pressure and volume 
overload of the right atrium. ${ }^{4-6}$ Although surgical approaches vary and patients often have had prior atrial incisions for their pre-Fontan operations or for vena caval cannulation, the ILT still requires an atrial incision that may result in damage to the sinus node or its artery and create the anatomic substrate for incisional atrial reentry tachycardia. In addition, the ILT subjects a part of the native right atrium to pressure overload, albeit to a lesser amount of atrial tissue compared with the APC. The ECC does not involve incisions near the sinus node or its artery or placement of incisions or sutures on the right atrial wall. The ECC aims to subject no part of the right atrium to pressure overload. Because of these factors, Giannico and colleagues $^{7}$ proposed the ECC as a way to reduce the incidence of arrhythmias in patients with single ventricles. Prior studies on this question have offered inconclusive results, mainly because of the lack of power from small study size.

A number of cross-sectional studies have reported that the ECC is associated with fewer arrhythmias. ${ }^{8-13}$ The total number of patients enrolled in these studies have ranged from $51^{12}$ to $165 .^{11}$ These studies showed an increased incidence of $\mathrm{SND}^{8-13}$ early postoperative SVT, ${ }^{8,9,12}$ and late-onset SVT $^{8,9,12}$ in the ILT cases compared with ECC cases.

Evidence that the ECC may be associated with worse SND primarily comes from 2 studies from a single center $^{14,15}$ that initially reported 36 and then 70 patients. They reported a higher incidence of SND with the ECC in both the early and late postoperative periods.

Third, a few studies have shown no significant difference in arrhythmia prevalence between ILT and ECC. ${ }^{6,16,17}$ Most noteworthy among these was a multicenter cross-sectional study of 520 patients from the Pediatric Heart Network Investigators. ${ }^{6}$ Their cohort consisted of 306 ILT and 69 ECC cases. They could see no difference in the time-adjusted incidence of clinical SVT between the ILT and the ECC.

To date, the only prospective study on this subject has been by Cohen and colleagues. ${ }^{17}$ The examined the incidence of arrhythmia on ECG and Holter monitoring between ECC and ILT using ECG and Holter monitoring. The found an incidence of SND of 13\% after both ECC and the ILT.

In another study from the Pediatric Heart Network, Blaufox and colleagues ${ }^{18}$ showed that a lower resting heart rate is associated with better functional outcome in patients undergoing the Fontan. They speculated that a lower resting heart rate with consequent longer filling time may be beneficial in the Fontan circulation, which is known to be pre-load dependent. ${ }^{18}$

In contrast to these earlier smaller studies, our larger multiinstitutional study shows that early postoperative arrhythmias are more prevalent in patients receiving the ECC compared with the ILT and that long-term arrhythmia burden is not different between the 2 procedures. This study has the advantage of evaluating a large population across numerous centers, reducing the potential effects of institutional surgical and postoperative techniques and arrhythmia management preferences. The most important and troublesome arrhythmia in patients undergoing the Fontan is late-occurring SVT (mainly AFL and IART), which is a source of significant morbidity and mortality, and difficult to manage. The main attraction of the ECC was the hope that it would be associated with a lower incidence of late SVT. This study suggests that the perceived lower incidence of arrhythmias after the ECC is likely due to the shorter length of follow-up in ECC compared with the ILT.

Although this study could not elucidate why the ECC does not result in fewer arrhythmias despite its theoretic advantages, a number of possible reasons can be speculated. The underlying heart defect or operations performed at the pre-Fontan stage may be the true cause of arrhythmias, which are then "unmasked" by the Fontan physiology. Alternatively, the dissection involved in the area of the right atrium to create a space for the ECC conduit as it courses between the inferior vena cava and the pulmonary artery may be more arrhythmogenic than realized. Another possibility is that the ILT, which involves a patch extending from the superior to the inferior vena cava, acts in a manner similar to the Maze operation, thereby reducing its potential arrhythmogenicity lower than expected. Further work is needed to elucidate the causes of late SVT after the Fontan.

Our finding that SVT requiring future management will develop in up to $20 \%$ to $25 \%$ of patients receiving the ILT and ECC should give cause for concern to all clinicians caring for these patients. Strategies to prevent such arrhythmias and to manage patients with these arrhythmias will likely assume critical importance in the future.

\section{Study Limitations}

This study, despite its large size, suffers from the disadvantages of any retrospective data collection. As with most such studies, not all patients had complete data availability for statistical analysis. The follow-up duration in our study is relatively short. Because the length of follow-up has been an important determinant of arrhythmia development in this and prior studies, we plan to revisit this cohort in 5 to 10 years. Our study focused on clinical bradyarrhythmia and tachyarrhythmias. Only those arrhythmias that were treated were included. We did not use any age-specific cutoffs of heart rate on ECG or ambulatory ECG monitoring to diagnose an arrhythmia. Such a stringent definition of arrhythmia likely underestimates the incidence.

\section{CONCLUSIONS}

Our study found that the ECC does not confer any long-term arrhythmia advantage over the ILT. Early 
postoperative bradyarrhythmia was seen more often after the ECC than the ILT. Therefore, the decision on which type of Fontan to perform on a specific patient should be based on individual, patient-related hemodynamic factors and surgical center preferences, and not on a presumed reduction in arrhythmias.

The authors thank the Department of Pediatrics, Children's Hospital of Philadelphia, for providing funding for this study; Jan Weber, RN, for nurse coordination support; Xuemei Zhang, MS, for statistical support; and the Pediatric and Congenital Electrophysiology Society and its membership for their support.

\section{References}

1. Fontan F, Baudet E. Surgical repair of tricuspid atresia. Thorax. 1971;26: 240-8.

2. de Leval MR, Kilner P, Gewillig M, Bull C. Total cavopulmonary connection: a logical alternative to atriopulmonary connection for complex Fontan operations. Experimental studies and early clinical experience. J Thorac Cardiovasc Surg. 1988;96:682-95.

3. Marcelletti C, Corno A, Giannico S, Marino B. Inferior vena cava-pulmonary artery extracardiac conduit. A new form of right heart bypass. J Thorac Cardiovasc Surg. 1990;100:228-32.

4. Balaji S, Gewillig M, Bull C, de Leval MR, Deanfield JE. Arrhythmias after the Fontan procedure. Comparison of total cavopulmonary connection and atriopulmonary connection. Circulation. 1991;84(5 Suppl):III162-7.

5. Cecchin F, Johnsrude CL, Perry JC, Friedman RA. Effect of age and surgical technique on symptomatic arrhythmias after the Fontan procedure. Am J Cardiol. 1995; 76:386-91.

6. Stephenson EA, Lu M, Berul CI, Etheridge SP, Idriss SF, Margossian R, et al. Arrhythmias in a contemporary Fontan cohort: prevalence and clinical associations in a multicenter cross-sectional study. J Am Coll Cardiol. 2010; 56:890-6.
7. Giannico S, Corno A, Marino B, Cicini MP, Gagliardi MG, Amodeo A, et al. Total extracardiac right heart bypass. Circulation. 1992;86(5 Suppl):II110-7.

8. Azakie A, McCrindle BW, Van Arsdell G, Benson LN, Coles J, Hamilton RET, et al. Extracardiac conduit versus lateral tunnel cavopulmonary connections at a single institution: impact on outcomes. J Thorac Cardiovasc Surg. 2001;122: $1219-28$.

9. Nurnberg JH, Ovroutski S, Alexi-Meskishvili V, Ewert P, Hetzer R, Lange PE. New onset arrhythmias after the extracardiac conduit Fontan operation compared with the intraatrial lateral tunnel procedure: early and midterm results. Ann Thorac Surg. 2004;78:1979-88.

10. Bae EJ, Lee JY, Noh CI, Kim WH, Kim YJ. Sinus node dysfunction after Fontan modifications-influence of surgical method. Int J Cardiol. 2003;88:285-91.

11. Lee JR, Kwak J, Kim KC, Min SK, Kim WH, Kim YJ, et al. Comparison of lateral tunnel and extracardiac conduit Fontan procedure. Interact Cardiovasc Thorac Surg. 2007;6:328-30.

12. Sarkis V, Sreeram N, Trieschmann U, Ben Mime L, Bennink G. Comparison of arrhythmia incidence after the extracardiac conduit versus the intracardiac lateral tunnel Fontan completion. Int J Cardiol. 2011;146:258-9.

13. Ovroutski S, Dahnert I, Alexi-Meskishvili V, Nurnberg JH, Hetzer R, Lange PE Preliminary analysis of arrhythmias after the Fontan operation with extracardiac conduit compared with intra-atrial lateral tunnel. Thorac Cardiovasc Surg. 2001 49:334-7.

14. Dilawar M, Bradley SM, Saul JP, Stroud MR, Balaji S. Sinus node dysfunction after intraatrial lateral tunnel and extracardiac conduit Fontan procedures. Pediatr Cardiol. 2003;24:284-8.

15. Kumar SP, Rubinstein CS, Simsic JM, Taylor AB, Saul JP, Bradley SM. Latera tunnel versus extracardiac conduit Fontan procedure: a concurrent comparison. Ann Thorac Surg. 2003;76:1389-96.

16. Hakacova N, Lakomy M, Kovacikova L. Arrhythmias after Fontan operation: comparison of lateral tunnel and extracardiac conduit. J Electrocardiol. 2008; 41:173-7.

17. Cohen MI, Bridges ND, Gaynor JW, Hoffman TM, Wernovsky G, Vetter VL, et al. Modifications to the cavopulmonary anastomosis do not eliminate early sinus node dysfunction. J Thorac Cardiovasc Surg. 2000;120:891-900.

18. Blaufox AD, Sleeper LA, Bradley DJ, Breitbart RE, Hordof A, Kanter RJ, et al. Functional status, heart rate, and rhythm abnormalities in 521 Fontan patients 6 to 18 years of age. J Thorac Cardiovasc Surg. 2008;136:100-7. 